Dom. Cien., ISSN: 2477-8818

Vol. 4, núm. 3, julio, 2018, pp. 189-210

Propuesta de puente aplicando el método de diseño AASHTO LRFD para la ciudad de Manta.

\title{
Propuesta de puente aplicando el método de diseño AASHTO LRFD para la
} ciudad de Manta

\section{Proposal of bridge slab using the AASHTO LRFD design method for the city of Manta \\ Proposta de ponte de laje aplicando o método de projeto AASHTO LRFD para a cidade de Manta.}

\author{
Carlos G. Delgado-Castro ${ }^{\mathrm{I}}$ \\ carlosgdelgado@uleam.edu.ec \\ Robert A. Rodríguez-Rivas II \\ roberto.rodriguez@hotmail.com \\ Weelinton A. Verá-Ávila ${ }^{\text {III }}$ \\ weelinton.vera@gmail.com
}

Correspondencia: carlosgdelgado@uleam.edu.ec

Recibido: 14 de agosto de 2017 * Corregido: 05 de octubre de 2017 * Aceptado: 17 de noviembre de 2017

I. Magister en Gestión Ambiental, Ingeniero Civil, Capacitación Global CAPAGLOB S.A prevención de riesgos laborales, Manabí, Ecuador.

II. Docente de la Universidad Laica Eloy Alfaro de Manabí, Manta, Ecuador.

III. Docente de la Universidad Laica Eloy Alfaro de Manabí, Manta, Ecuador. 


\title{
Resumen
}

Es necesario mencionar, que Ecuador no cuenta con una normativa propia de carácter técnico y actualizado para el diseño de puentes vehiculares, por lo que ha sido necesario recurrir a normas de carácter internacional, siendo las normas americanas dada por la AASHTO la más utilizada. Con base en este planteamiento, se propone el diseño el método de AASHTO LRFD para la realización de puente losa en la ciudad de manta. La investigación siguió una metodología cuanli- cuantitativa. El enfoque epistémico fue la lógica racional. De carácter no experimental de campo y documental. La información fue analizada a través del análisis interpretativo de los datos aportados por la revisión documental de las características poblacionales y viales del cantón de Manta, por lo que genero la necesidad de proponer un proyecto de puente losa con miras a ser utilizado en otras ciudades con características similares a la estudiada. Entre sus conclusiones se puede mencionar que fue posible determinar que el correcto dimensionamiento de la estructura del puente losa, dependerá específicamente del proyecto que se esté llevando a cabo, considerando principalmente la longitud del puente, así como el peralte de la losa.

Palabras clave: puente losa normas;; normas LRFD y vía.

\begin{abstract}
It is necessary to mention, that Ecuador has a regulation itself of up-to-date and technical character for the design of vehicular bridges, so it has not been necessary to resort to international standards, being the American standards given by the AASHTO more used. Based on this approach, intends to design the AASHTO LRFD method for the realization of bridge slab in the city of manta. The investigation followed a methodology cuanli-cuntitativa. The epistemic approach was rational logic. Non-experimental character of field and documentary. The information was analyzed through the interpretative analysis of data provided by the document review of population and road characteristics of the canton of blanket, by what genre the need to propose a project of bridge slab to be used in other cities with similar characteristics to the studied. Its conclusions include that it was possible to determine the correct sizing of the structure of bridge slab, will depend specifically on project that is being carried out, mainly considering the length of the bridge as well as the can't of the slab.
\end{abstract}

Keywords: bridge slab; AASHTO standards; standards LRFD and via. 


\section{Resumo}

Deve ser mencionado que o Equador não tem regulamentos de design técnico e específico atualizados para o personagem pontes veiculares, por isso foi necessário recorrer a padrões internacionais, padrões americanos sendo dado pela AASHTO mais frequentemente usado. Com base nessa abordagem, propõe-se o desenho do método AASHTO LRFD para a realização de uma ponte de laje na cidade de manta. A pesquisa seguiu uma metodologia quantitativa-quantitativa. A abordagem epistêmica foi a lógica racional. De caráter não experimental de campo e documentário. Os dados foram analisados através da análise interpretativa dos dados fornecidos pela análise do documento da população e rodoviárias características do cantão de Manta, então eu gerar a necessidade de propor uma ponte projecto de laje com vista a ser utilizado em outras cidades características semelhantes à estudada. Entre as suas conclusões podem ser mencionados que foi possível determinar o dimensionamento correcto da estrutura de ponte da laje, o projecto dependerá especificamente a ser realizada, especialmente considerando o comprimento da ponte, bem como a profundidade da laje.

Palavras chave: normas de laje de ponte; AASHTO; Padrões LRFD e via.

\section{Desarrollo}

El cambio más significativo que introdujo la norma AASHTO (1996) en el cálculo de estructuras de puentes es la utilización del método LRFD: Load and Resistance Factor Design, el cual corresponde, según Ochoa (2008), a un método de estados límites últimos, que considera factores para cada carga y para cada resistencia nominal. Otro aspecto importante que introdujo esta norma es la forma de combinar las cargas, ya que ésta considera algunos factores que van a cambiar el margen de seguridad del puente, dando una mayor confiabilidad a la estructura. El mismo Disposiciones de la norma AASHTO LRFD. Otro aspecto importante que introdujo esta norma es la forma de combinar las cargas, ya que según Perales (2013) ésta considera algunos factores que van a cambiar el margen de seguridad del puente, dando una mayor confiabilidad a la estructura.

El mismo autor, indica que estos factores corresponden a coeficientes de ductilidad, redundancia e importancia de la estructura. Esta norma, indica Nilson (2011) introdujo una nueva forma de combinar el modelo de carga vehicular, debido a que se establecen tres tipos de cargas vivas vehiculares: Camión de Diseño, Tándem de Diseño y Carga de Faja de Diseño. En general, a 
Propuesta de puente aplicando el método de diseño AASHTO LRFD para la ciudad de Manta.

continuación, se muestran las disposiciones más importantes de la norma AASHTO que se utilizarán en el posterior diseño de las superestructuras de los modelos elegidos.

Estados límites (AASHTO LRFD). Un Estado Límite define condiciones que se quiere que una estructura satisfaga. En general, un elemento estructural tendrá que satisfacer diferentes estados límites. Los Estados Límites definidos por la norma AASHTO (2004) LRFD son los que se presentan a continuación:

Estado Límite de Servicio. Se debe considerar como restricciones a las tensiones, deformaciones y anchos de fisura, bajo condiciones regulares de servicio.

Estado Límite de Fatiga y Fractura. Este estado límite se debe tomar como restricciones asignadas al rango de esfuerzos. El estado límite de fractura apunta Escalante (2014) se debe considerar como un conjunto de requisitos sobre resistencia de materiales de las Especificaciones sobre los materiales. La combinación de carga de este estado límite es la siguiente.

Estado Límite de Resistencia. Félix (2015), explica que el estado límite de resistencia define los criterios de capacidad última de la estructura para asegurar su resistencia y estabilidad, tanto local como global, para resistir las combinaciones de carga estadísticamente significativas que se espera que el puente experimente en su vida útil.

Estados Límites correspondientes a Eventos Extremos. Es importante considerar el estado límite que corresponde a eventos extremos para asegurar la vida útil estructuralmente hablando de un puente durante lluvias intensas que produzcan inundaciones o sismos de grados significativos, o por ejemplo cuando es impactado por un elemento externo, posiblemente en situaciones con socavación.

\section{Cargas (AASHTO LRFD)}

Cargas Permanentes (AASHTO LRFD). De acuerdo a la norma (AASHTO) se distinguen dos grupos: Cargas Permanentes (AASHTO LRFD) y Cargas de Suelo (AASHTO LRFD).

Cargas Transitorias (AASHTO LRFD). Según la norma (AASHTO, 2004) se definen las siguientes cargas transitorias a utilizar en esta memoria son:

DC: Carga permanente de componentes estructurales

DW: Carga permanente de superficies de rodamiento

IM: Carga Dinámica

LL: Carga Viva vehicular

Carga Viva (AASHTO LRFD). La carga viva vehicular consiste en el peso de las cargas en movimiento sobre el puente. Se tienen dos tipos: Vehicular y Peatonal. 
Carga peatonal (AASHTO LRFD). La carga peatonal consiste en una carga de 0.0036 (MPa) que se aplica a todos los pasillos de más de 600( $\mathrm{mm})$, y que se considera simultáneamente con la carga vehicular. Los puentes utilizados solamente para tránsito peatonal y/o de bicicletas serán diseñados para una carga viva de $0.0041(\mathrm{MPa})$

Carga Vehicular (AASHTO LRFD). Según la norma LFRD de la (AASHTO) el número de vías de tránsito que se debe fijar, es la parte entera del cociente: w/3.6.

Se definen tres tipos de cargas: Carga de Camión, Carga de Faja y Carga de Tándem. La carga vehicular total a utilizar es designada "Carga HL-9". Esta carga será la mayor solicitación proveniente de las combinaciones:

-Carga de Camión + Carga de faja (Utilizada en nuestro país)

-Carga de Tándem + Carga de Faja

Carga de Camión (AASHTO LRFD2): El camión de diseño de la norma (AASHTO) LRFD es el HL-93 similar al camión HS 20 - 44 especificado en la norma Standard. Transversalmente, el ancho de vía de diseño es de $3.6(\mathrm{~m})$, con una separación entre ejes de ruedas de 1,8(m), tal como se muestra en la siguiente figura.

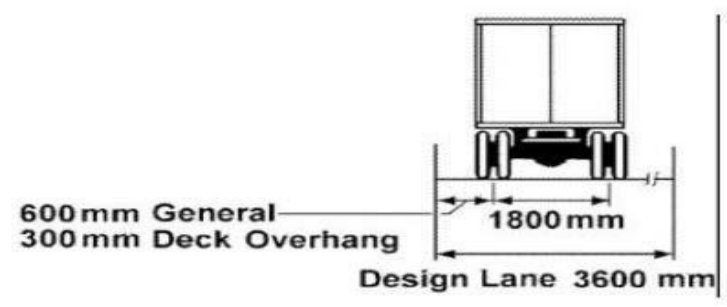

Figura 1. Sección transversal del Camión de la norma AASHTO LRFD

\section{Fuente: AASHTO LRFD}

En la dirección longitudinal se tiene una distancia de separación entre ejes de ruedas de $4.3(\mathrm{~m})$ y otra que varía entre $4.3(\mathrm{~m})$ y $9.0(\mathrm{~m})$, considerando la que provoque las máximas solicitaciones. Los dos últimos ejes reciben la mayor parte de la carga, 14,8 (T) en cada eje, y 3.57 (T) en su eje delantero, lo que se aprecia en la siguiente figura: 
Propuesta de puente aplicando el método de diseño AASHTO LRFD para la ciudad de Manta.

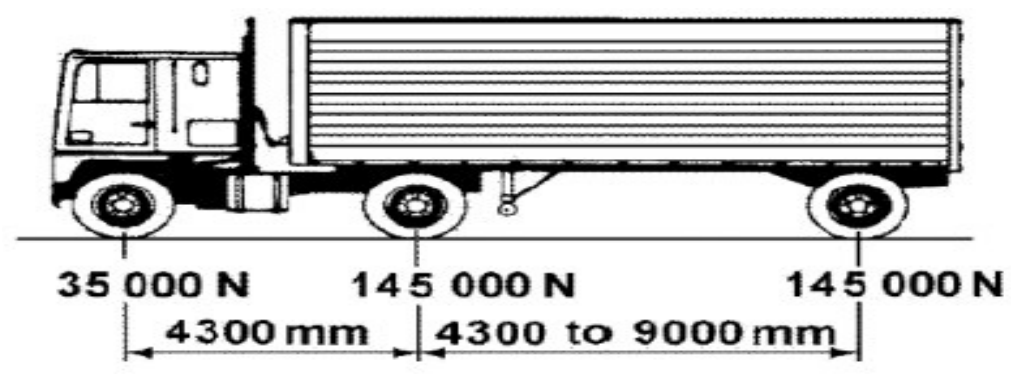

Figura 2. Sección longitudinal del Camión de la norma AASHTO LRFD

Fuente: AASHTO LRFD

Carga de Tándem (AASHTO LRFD). El Tándem de diseño consiste en un par de ejes de carga igual a $11.22(\mathrm{~T})$ espaciados longitudinalmente a $1.2(\mathrm{~m})$. El espaciamiento transversal de ruedas

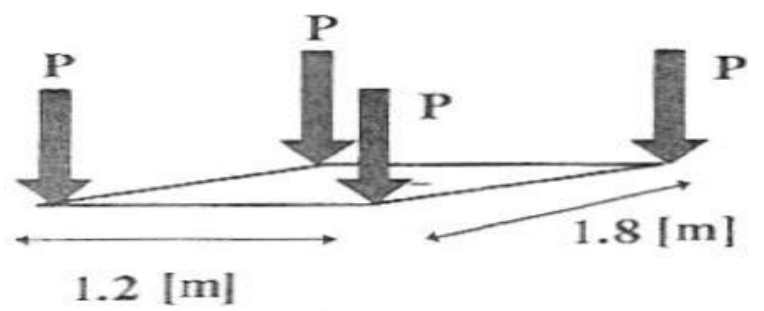

será de $1.8(\mathrm{~m})$.

Figura3. Carga de Tándem

Fuente: AASHTO LRFD

Según el NEVI 12 (Norma Ecuatoriana Vial), no hace ninguna diferenciación entre los camiones de diseño para los dos métodos, es decir establece un solo camión de diseño, por lo que se adoptará las características del camión para ambos métodos.

Carga de faja (AASHTO LRFD). La carga de faja consiste en una carga de 0.949 (T/m), distribuida uniformemente en la dirección longitudinal. Transversalmente, la carga de faja se asume uniformemente distribuida sobre un ancho de $3(\mathrm{~m})$.

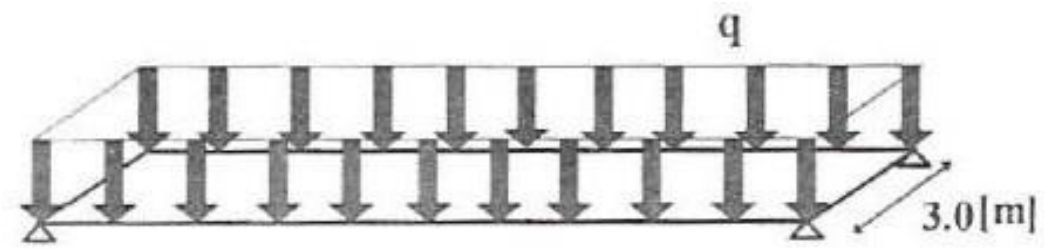

Figura 4. Carga de faja de la norma AASHTO LRFD

Fuente: AASHTO LRFD

\section{Carga de fatiga (AASHTO LRFD)}


Según (AASHTO, 2004) la carga móvil de fatiga consiste en un camión igual al establecido en el artículo 1.4.6.4.3.2.1 del presente proyecto, solo que con un espaciamiento fijo de 9,0 (m) entre ejes de peso igual a 14.8 (T). Además, no se considera la carga de faja. El estado de fatiga no necesita ser investigado para las losas de hormigón con vigas múltiples.

\section{Carga dinámica admisible IM (AASHTO LRFD)}

Los efectos estáticos del camión o tándem de diseño serán aumentados en el porcentaje.

Tablas 1. Carga dinámica admisible

\begin{tabular}{lc}
\hline \multicolumn{1}{c}{ Componentes } & IM \\
& $(\mathbf{\%})$ \\
\hline Justas de losa - todos los estados limites & 75 \\
\hline Todos los demás componentes & 15 \\
\hline Estado límite de la fatiga y fractura & 33 \\
\hline Todos los demás estados limites & \\
\hline
\end{tabular}

Fuente: AASHTO LRFD

El factor que se aplicará a la carga estática será: $\mathrm{C}=(\mathrm{I}+1 \mathrm{~m} / 100)$. La carga dinámica admisible no se aplica sobre la carga peatonal ni sobre la carga de faja. Aplicación de las cargas de camión. La carga vehicular a utilizar se determina como la condición más desfavorable entre: la carga de camión más la carga de faja o la carga de tándem más la carga de faja. En el diseño AASHTO LFRD, la carga de camión o de tándem deberá posicionarse transversalmente, tal que el centro de la rueda externa este a más de: 0,3(m) de la cara interna del guardarruedas o de la baranda, para el diseño de la losa en voladizo y de $0,6(\mathrm{~m})$ del borde de la vía de diseño, para el diseño de todos los demás elementos.

\section{Metodología}

La investigación siguió una metodología cuanli- cuantitativa. El enfoque epistémico fue la lógica racional. De carácter no experimental de campo y documental. Considerada de campo porque se realizó en el lugar en donde se dio el problema y se estudiaron en el mismo lugar las posibles soluciones para dar la repuesta al problema y documental ya que se analizó la información descrita sobre el tema para así establecer un juicio del cual se partió para estudiar el comportamiento seguridad estructural de Puentes Vehiculares mediante el análisis de las dos filosofías de diseño. La 
Propuesta de puente aplicando el método de diseño AASHTO LRFD para la ciudad de Manta.

información fue analizada a través del análisis interpretativo de los datos aportados por la revisión documental de las características poblacionales y viales del cantón de Manta.

\section{Resultados}

Posterior al análisis interpretativo de los datos aportados por la revisión documental de las características poblacionales y viales del cantón de Manta, se posible proponer, un método de diseño AASHTO LRFD para la ciudad de Manta, que además de beneficiar a sus habitantes, de igual manera a los ingenieros de la localidad al ser capacitados en este tipo de diseño de puente losa. La propuesta puede ser inicialmente ejecutada en la ciudad de Manta, pero se pretende que los talleres que se impartan alcancen otros cantones pertenecientes a la provincia de Manabí, con la finalidad de que se difunda de forma oportuna en el País. La misma se presenta a continuación: Objetivo general de la propuesta: proponer el proyecto de un puente losa aplicando el método de diseño AASHTO LRFD.

\section{Objetivos específicos:}

Generar un análisis del método AASHTO LRFD.

Diseñar el grafico de armadura de la losa por medio del método AASHTO LRFD

Socializar los datos obtenidos por medio de la aplicación del método de diseño AASHTO LRFD a través de la generación de un puente losa.

Diseño de un puente losa mediante el método AASHTO LRFD.

\begin{tabular}{cc}
\hline Descripción & Medida \\
\hline Resistencia del concreto $\left(f^{\prime} c\right)$ & $280 \mathrm{Kg} . / \mathrm{cm}$ \\
\hline Fluencia del acero $\left(\mathrm{f}^{\prime} \mathrm{y}\right)$ & $4200 \mathrm{Kg} . / \mathrm{cm}^{2}$ \\
\hline Carpeta asfáltica & $0,05 \mathrm{~m}$ \\
\hline Densidad del concreto & $2400 \mathrm{Kg} . / \mathrm{cm}^{3}$ \\
\hline Densidad del asfalto & $2250 \mathrm{Kg} . \mathrm{cm}^{3}$ \\
\hline Longitud libre & $10 \mathrm{~m}$ \\
\hline Ancho de la calzada & $9,2 \mathrm{~m}$ \\
\hline Peso del pretil & $0,834 \mathrm{Tn} / \mathrm{m}$ \\
\hline Carga del carril & $0,95 \mathrm{Tn} / \mathrm{m}$
\end{tabular}

Tabla 2. Datos generales para el diseño de Puente Losa. 
Propuesta de puente aplicando el método de diseño AASHTO LRFD para la ciudad de Manta.

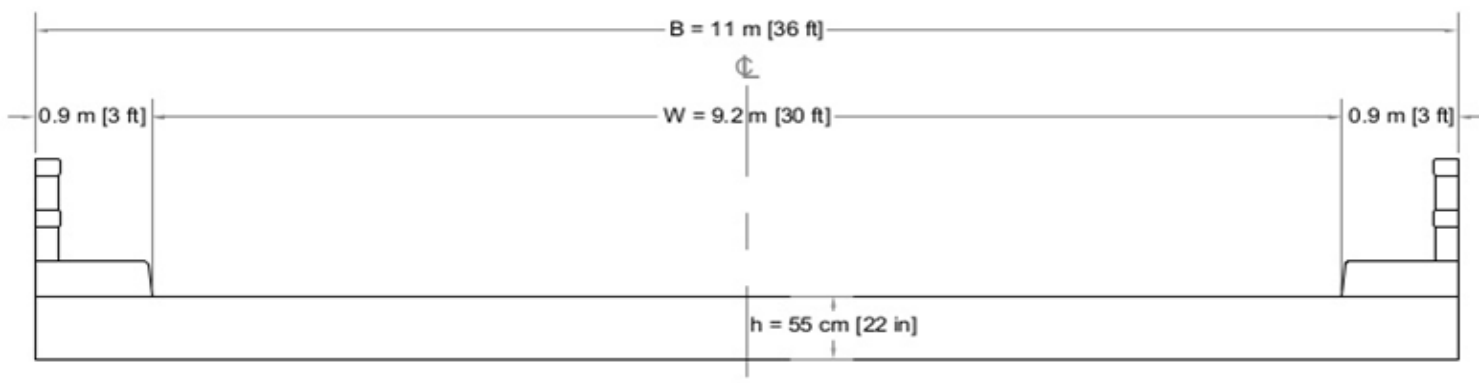

Fuente: Elaboración propia

Figura 5. Puente Losa, $L U Z=10 \mathrm{~m}$ y $A$ ncho $=9.2 \mathrm{~m}$ Corte longitudinal.

Fuente: Elaboración propia

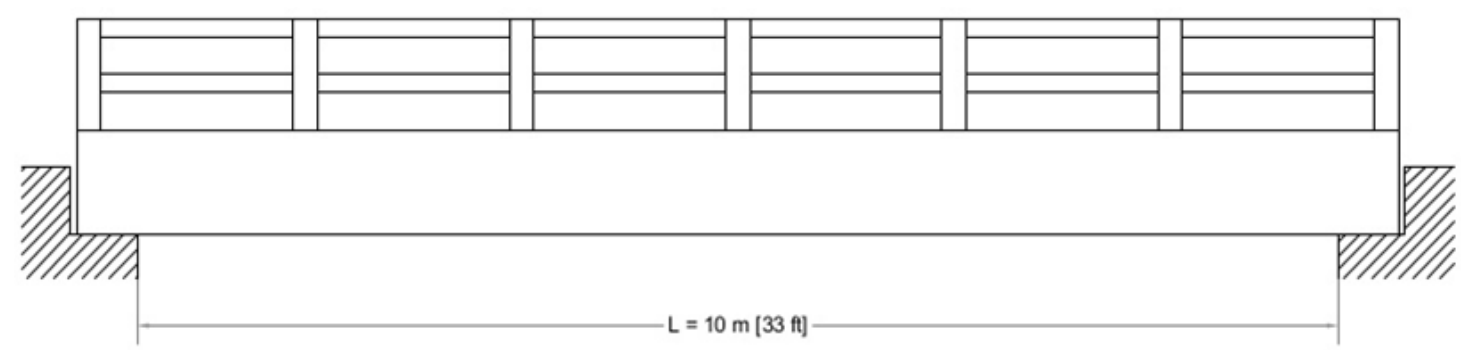

Figura 6. Puente Losa, $L U Z=10 \mathrm{~m}$ y Ancho $=9.20 \mathrm{~m}$ Corte transversal.

Fuente: Elaboración propia.

\section{Para el análisis, la losa se divide en franjas de dos tipos:}

Interiores

De borde

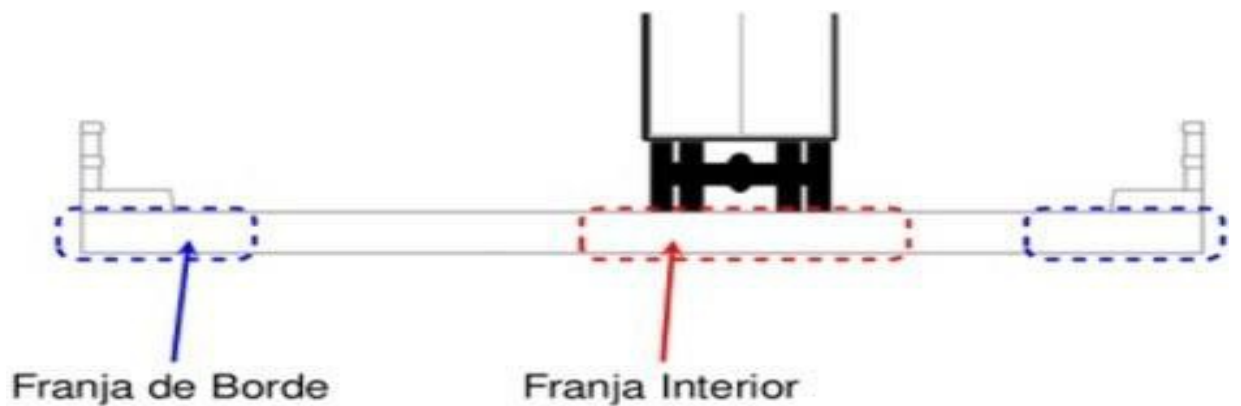


Propuesta de puente aplicando el método de diseño AASHTO LRFD para la ciudad de Manta.

Figura 7. Comportamiento bajo el peso del camión.

Fuente: Elaboración propia.

\section{Pre dimensionamiento del Peralte}

$$
h=\frac{1,2(10+3)}{30}=0,52 \mathrm{~m} \text { Se adoptará una losa de } \mathrm{h}=0,55 \mathrm{~m}
$$

Calculo de franjas interiores, franjas de borde y cargas muertas para el diseño de puente losa AASHTO LRFD.

Ancho de Franjas

\begin{tabular}{|c|c|c|c|c|}
\hline & & MKS & USC & \\
\hline $\begin{array}{l}\text { Un carril } \\
\text { cargado }\end{array}$ & 흘 SLL & $\begin{aligned} E & =\left(3+5 \sqrt{L^{\prime} B^{\prime}}\right) / 12 \\
B^{\prime} & =\min \{9 \mathrm{~m}, B\} \\
L^{\prime} & =\min \{18 \mathrm{~m}, L\}\end{aligned}$ & $\begin{aligned} E & =\left(10+5 \sqrt{L^{\prime} B^{\prime}}\right) / 12 \\
B^{\prime} & =\min \{30 \mathrm{tt}, B\} \\
L^{\prime} & =\min \{60 \mathrm{tt}, L\}\end{aligned}$ & $(4.6 .2 .3-1)$ \\
\hline Múltiples & $\begin{array}{l}\overline{\frac{\pi}{c}} \\
\text { 荧 } \\
M L L\end{array}$ & $\begin{aligned} E & =2.10+0.12 \sqrt{L^{\prime} B^{\prime}} \leq \frac{B}{N_{L}} \\
B^{\prime} & =\min \{18 \mathrm{~m}, B\} \\
L^{\prime} & =\min \{18 \mathrm{~m}, L\}\end{aligned}$ & $\begin{aligned} E & =7+0.12 \sqrt{L^{\prime} B^{\prime}} \leq \frac{B}{N_{L}} \\
B^{\prime} & =\min \{60 \mathrm{ft}, B\} \\
L^{\prime} & =\min \{60 \mathrm{ft}, L\}\end{aligned}$ & $(4.6 .2 .3-2)$ \\
\hline $\begin{array}{l}\text { carriles } \\
\text { cargados }\end{array}$ & 突 ㅎํㄴ & $E_{Q}=\min \left\{\begin{array}{l}E / 4+E_{\text {prest }}+0.3 \mathrm{~m} \\
E / 2 \\
1.80 \mathrm{~m}\end{array}\right.$ & $E_{e}=\min \left\{\begin{array}{l}E / 4+E_{\text {pretl }} \\
E / 2 \\
6 \pi\end{array}\right.$ & $\begin{array}{l}-1 \mathrm{ft} \\
(4.6 .2 .1 .4)\end{array}$ \\
\hline
\end{tabular}

Figura 8. Ancho de franja interior y franja de borde del puente.

Fuente: AASHTO LRFD

\section{Franja Interior}

Tabla 3. Datos de la franja interior del Puente Losa.

Un carril cargado Múltiples carriles cargados

\begin{tabular}{r|r}
$\mathrm{B}^{`}=9 \mathrm{~m}$ & $\mathrm{~B}{ }^{`}=11 \mathrm{~m}$ \\
$\mathrm{~L}=10 \mathrm{~m}$ & $\mathrm{~L}=10 \mathrm{~m}$ \\
$\mathrm{E}=4,20 \mathrm{~m}$ & $\mathrm{E}=3,36 \mathrm{~m}<\mathrm{B} / \mathrm{Nvía}$ \\
& $\mathrm{E}=3,36 \mathrm{~m}<3,67 \mathrm{~m}$
\end{tabular}


Propuesta de puente aplicando el método de diseño AASHTO LRFD para la ciudad de Manta.

Fuente: Elaboración propia.

Nuestra franja interior definitiva será el menor valor de "E" entre un carril cargado y múltiples carriles cargados, es decir: $\mathrm{E}=3,36 \mathrm{~m}$

Franja de borde: 1,80 m. Nuestra franja de borde será el menor de los 3 valores anteriores, es decir: $\mathrm{E}=1,68 \mathrm{~m}$.

Tabla 4. Cargas muertas de la losa Franja Interior

\begin{tabular}{|c|}
\hline $\mathrm{E} / 4+\mathrm{Ep}+0.3=2.04 \mathrm{~m}$ \\
\hline $\mathrm{E} / 2=1,68$ \\
\hline $180 \mathrm{~m}$ \\
\hline
\end{tabular}

Fuente: Elaboración propia.

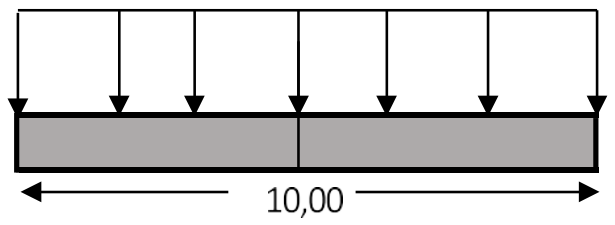

Figura 9. Cargas muertas de la losa Franja Interior

Franja de Borde

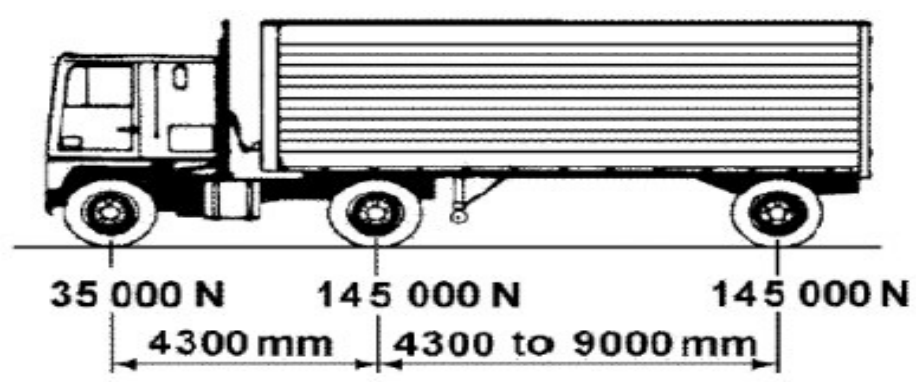

Calculo de carga viva para el diseño de puente.

Figura 10. Camión de Diseño HL-93

Fuente: Elaboración propia

Cargas Vivas (LL+IM). Para puentes simplemente apoyados y cargas HL-93 Momentos por cargas vivas

Tabla 5. Cargas vivas según su longitud 
Propuesta de puente aplicando el método de diseño AASHTO LRFD para la ciudad de Manta.

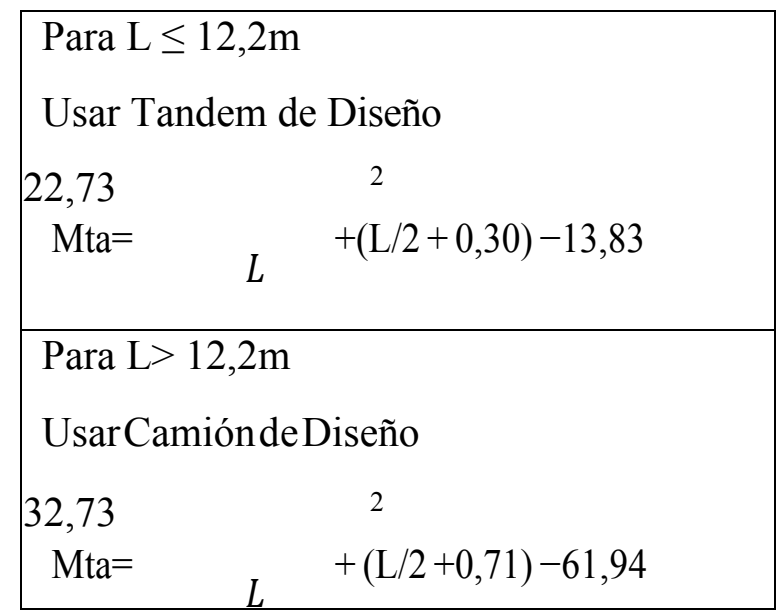

Fuente: Elaboración propia

Como $\mathrm{L}=10 \mathrm{~m} \leq 12,2 \mathrm{~m}$; usamos un Mta--

Utilizamos la carga de carril que es Wllaa =0,95 TT/m. El momento causado por esta carga en el centro del claro es

$M L a=W L a * \mathrm{~L} 2 / 8=11,88 \mathrm{Tn}-\mathrm{m}$. El impacto es de $\mathrm{IM}=33 \%$

FRANJA INTERIOR: Es decir la carga viva + impacto + carga de carril es:

$\mathrm{MLL}+\mathrm{IM}=(1+0,33) * 50,02+11,88=78,40$ Tn-m.

FRANJA DE BORDE. Debido a que un camión completo no cabe en una franja de borde se utiliza solo la mitad de su carga, es decir $\mathrm{DF}=0,5$

$$
\mathrm{M} 2 \mathrm{LL}+\mathrm{IM}=0,5(78.40)=39,20 \mathrm{Tn}-\mathrm{m}
$$

\section{Resumen de cargas}

Tabla 6. Resumen de cargas

\begin{tabular}{|c|r|r|}
\hline & F.Interior & F. de Borde \\
\hline DC & 55,42 Tn-m & 38,13 Tn-m \\
\hline DW & 4,72 Tn-m & 1,10 Tn-m \\
\hline LL+IM & 78,40 Tn-m & $39,20 \mathrm{Tn}-\mathrm{m}$ \\
\hline
\end{tabular}


Propuesta de puente aplicando el método de diseño AASHTO LRFD para la ciudad de Manta.

Fuente: Elaboración propia

\section{Factores de Carga}

Tabla 7. Cargas vivas según su longitud

\begin{tabular}{|c|c|c|}
\hline & Resistencia & Servicio \\
\hline DC & 1,25 & 1,00 \\
\hline DW & 1,50 & 1,00 \\
\hline $\mathbf{L L}+\mathbf{I M}$ & 1,75 & 1,00 \\
\hline
\end{tabular}

Fuente: AASHTO LRFD

A continuación, para encontrar el momento último de resistencia y momento último de servicio multiplicamos las cargas encontradas anteriormente por su respectivo factor de carga, obteniendo lo siguiente:

Tabla 8. Momentos de resistencia y momentos de servicio.

\begin{tabular}{|r|r|r|c|}
\hline & F. Interior & F.deBorde & F.de Carga \\
\hline DC & $55,42 \mathrm{Tn}-\mathrm{m}$ & $38,13 \mathrm{Tn}-\mathrm{m}$ & x 1,25 \\
\hline DW & $4,72 \mathrm{Tn}-\mathrm{m}$ & $1,10 \mathrm{Tn}-\mathrm{m}$ & x 1,50 \\
\hline LL + IM & $78,40 \mathrm{~T}-\mathrm{nm}$ & $39,20 \mathrm{Tn}-\mathrm{m}$ & X 1,75 \\
\hline M.u (Resistencia) & $\mathbf{2 1 3 , 5 5}$ Tn-m & $\mathbf{1 1 7 , 9 1}$ Tn-m & \\
M.s(Servicio) & $\mathbf{1 3 8 , 5 4}$ Tn-m & $\mathbf{7 8 , 4 3}$ Tn-m & \\
& & & \\
& & &
\end{tabular}

Fuente: Elaboración propia

\section{Resistencia}

Factor de resistencia a flexión requerido

$\mathrm{R}=\mathrm{Mu} / \phi^{*} \mathrm{~b} * \mathrm{~d} 2$ 
Propuesta de puente aplicando el método de diseño AASHTO LRFD para la ciudad de Manta.

Necesitamos probar con un diámetro de varilla ya que requerimos conocer el ancho efectivo (d).

Probaremos con una varilla $24 \mathrm{~mm}$

El recubrimiento inferior requerido para losas coladas en sitio es de $2,50 \mathrm{~cm}$

$d=(55 \mathrm{~cm}-2,5 \mathrm{~cm}-2,4 \mathrm{~cm} / 2)$

$\mathrm{d}=51,30 \mathrm{~cm}$

\section{Franjas interiores}

$$
\begin{aligned}
& \mathrm{R}=\underset{\mathrm{Mu}}{\emptyset * b * \mathrm{~d} 2} \\
& \rho=0,85 \\
& A \mathrm{~s}=\rho * \mathrm{~b} * d \\
& A \mathrm{~s}=0,007 * 100 \mathrm{~cm} * 51,30 \mathrm{~cm}=34,88 \mathrm{~cm} 2 \\
& 100 * \text { área nom de la varilla } \\
& 100 * \frac{24 * 24 * 3,1416}{400} \\
& S=\frac{34,88}{}
\end{aligned}
$$

Usaremos varilla de 24mm @ 13cm para las franjas interiores

\section{Franjas de borde}

$$
\begin{aligned}
& A \mathrm{~s}=\rho * \mathrm{~b} * d \\
& A \mathrm{~s}=0,008 * 100 \mathrm{~cm} * 51,30 \mathrm{~cm}=38,88 \mathrm{c} \mathrm{m} 2
\end{aligned}
$$

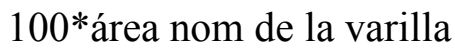

$$
\begin{aligned}
& 100 * \frac{24 * 24 * 3,1416}{400} \\
& 38,88
\end{aligned}
$$

Usaremos varilla de 24mm @ 12cm para las franjas exteriores 
Propuesta de puente aplicando el método de diseño AASHTO LRFD para la ciudad de Manta.

Tabla 9. Datos para poder calcular el esfuerzo limite en el acero.

\begin{tabular}{|c|r|}
\hline \multirow{2}{*}{ ye } & Factor de exposición \\
\cline { 2 - 3 } & Recubrimiento de concreto desde la fibra extrema a tensión hasta el \\
& centro del esfuerzo más cercano \\
\hline \multirow{2}{*}{$d d_{c c}$} & 0,75 para condición expuesto al agua \\
\hline $\mathrm{s}$ & Espaciamiento de varillas \\
\hline $\mathrm{h}$ & Espesor o altura del elemento \\
\hline
\end{tabular}

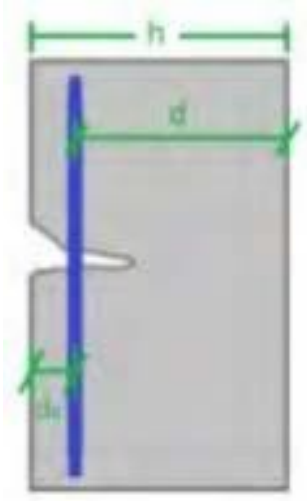

Fuente: Elaboración propia

Figura 11. Simulación del agrietamiento en el puente

Fuente: Elaboración propia

Procedemos al cálculo: 
Propuesta de puente aplicando el método de diseño AASHTO LRFD para la ciudad de Manta.

$$
\begin{gathered}
\mathrm{dc}=h-\mathrm{d} \quad \mathrm{dc}=55 \mathrm{~cm}-51,30 \mathrm{~cm}=3,70 \mathrm{~cm} \\
\beta_{S}=1+\frac{3,70 \mathrm{~cm}}{0,7(55 \mathrm{~cm}-3,70 \mathrm{~cm})}
\end{gathered}
$$

\section{Franjas Interiores:}

$$
\text { Fss }=\frac{125 * 1}{1,1(13 \mathrm{~cm}+2(3,70 \mathrm{~cm})}
$$

Fss $=5,56 \mathrm{Tn} / \mathrm{cm}^{2}$

Escogemos el menor, por lo tanto:

Fss $=2,52 \mathrm{Tn}$

\section{Franjas de borde:}

$$
\begin{gathered}
\mathrm{F}_{S S}=\frac{125 * 1}{1,1(12 \mathrm{sm}+2(3,70 \mathrm{sm})} \\
\mathrm{F}_{S S}=5,84 \mathrm{Tn} / \mathrm{cm}^{2}
\end{gathered}
$$

Escogemos el menor, por lo tanto:

$$
\text { Fss }=2,52 \mathrm{Tn}
$$

Entonces este valor límite tenemos que compararlo con nuestro esfuerzo que existe en nuestra sección.

\section{Esfuerzo en el Acero}

\section{Sección elástica agrietada (Condición de servicio)}
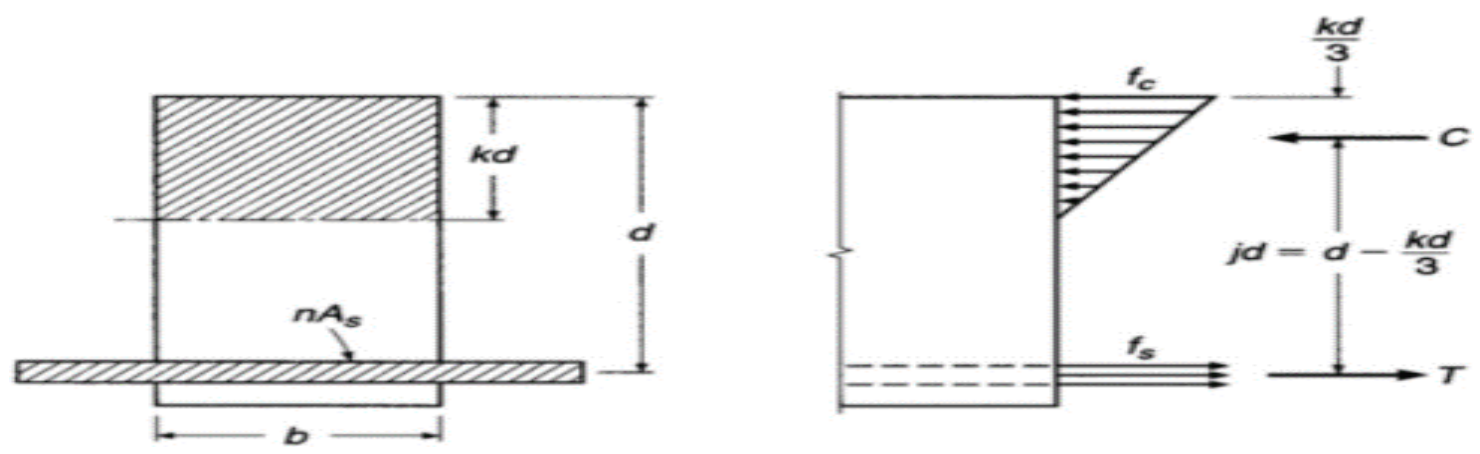

Figura 12. Esfuerzo en el Acero - Sección elástica

Fuente: Elaboración propia (2018) 
Propuesta de puente aplicando el método de diseño AASHTO LRFD para la ciudad de Manta.

\section{Calculamos la relación modular:}

\section{Donde:}

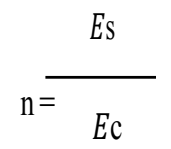

$$
\begin{aligned}
& E s=2000.00 \mathrm{Tn} / \mathrm{cm} 2 \\
& E c=267.73 \mathrm{Tn} / \mathrm{cm} 2
\end{aligned}
$$

Módulo de elasticidad del acero

Módulo de elasticidad del concreto EEee $=16000 \sqrt{ } F^{\prime}$ ee

$$
\mathrm{n}=\frac{2000}{267.73}=7.47
$$

\section{Franjas Interiores}

La cuantía de acero es:

$\rho=0.007(24 \mathrm{~mm} @ 12 \mathrm{~cm}) \operatorname{con} \mathrm{As}=34.88 \mathrm{~cm} 2$

$$
\begin{gathered}
k=\sqrt{ }(p n)^{2}+p n-p n \\
k=\sqrt{ } 0.007 * 7.47)^{2}+(0.007 * 7.47)-(0.007 * 7.47) \\
k=0.18 \\
j=1-\mathrm{k} / 3 \quad j=1-0.18 / 3 \quad j=0.94
\end{gathered}
$$

Con esto ya podemos calcular el esfuerzo en el acero a tensión:

$$
\begin{aligned}
& \mathrm{Fs}=\underline{M s} \\
& \text { As * E*jd } \\
& (138.54 * 100) \\
& \mathrm{Fs}=(138.54 * 100) \\
& (34.88 * 3.36) * 0.94 * 51.30 \\
& \text { Fs }=2.45 \\
& \mathrm{Fs}=2.45 \leq \mathrm{Fss}_{s}=2.52
\end{aligned}
$$

Si cumple esfuerzo en el acero a Tensión Esfuerzo límite. 
Propuesta de puente aplicando el método de diseño AASHTO LRFD para la ciudad de Manta.

\section{Franjas de Borde}

La cuantía de acero es:

$$
\begin{gathered}
\rho=0.008(24 \mathrm{~mm} @ 12 \mathrm{~cm}) \operatorname{con} \mathrm{As}=\frac{38.80 \mathrm{~cm} 2}{k=\sqrt{(p n)^{2}+p n-p n}} \\
k=\sqrt{ }(0.008 * 7.47)^{2}+(0.008 * 7.47)-(0.008 * 7.47) \\
k=0.19 \\
j=1-\mathrm{k} / 3 \\
j=1-0.19 / 3 \\
j=0.94
\end{gathered}
$$

Con esto ya podemos calcular el esfuerzo en el acero a tensión:

$$
\begin{gathered}
\mathrm{Fs}=M s \\
\frac{A s * E * j d}{(78.43 * 100)} \\
\mathrm{Fs}=(38.80 * 1.68) * 0.94 * 51.30 \mathrm{Fs}
\end{gathered}=2.50
$$

Fs $=2.50 \leq$ Fss $=2.52 \square$ Si cumple Esfuerzo en el acero a Tensión Esfuerzo límite.

\section{Refuerzo Distribuido}

El Refuerzo distribuido sirve para distribuir los momentos al refuerzo principal

\section{\% de Refuerzo Distribuido del Refuerzo Principal}

$\%$ Ref. Distrib. $=\underline{55.2 \leq 50 \%}$

$\sqrt{ } L$

Para nuestro caso obtenemos:

$$
\% \text { REf. Distribo. }=\frac{55,2}{\sqrt{ } 10}=17.46 \% \leq 50 \%
$$

Usando un As $=37.62 \mathrm{~cm} 2$ como un área de acero promedio del Refuerzo Principal de las franjas interiores y exteriores, tenemos lo siguiente: 
Propuesta de puente aplicando el método de diseño AASHTO LRFD para la ciudad de Manta.

Astemp $=17.46 \%$

$$
100 * 37.62=6.50 \mathrm{~cm}
$$

Separación=30 cm Usaremos varilla de 16 mm@30 cm

\section{Refuerzo Mínimo}

\section{Refuerzo por temperatura y retracción de fraguado}

${ }_{\mathrm{g}} \mathrm{A}_{\mathrm{S}}$ Temp. $\underline{\mathbf{7 , 8 2}}$

Fy

$\mathrm{A}_{\mathrm{S}}$ Temp $7,82 \underline{55 * 100}=10.24 \mathrm{~cm} 2$

$$
4200
$$

Separación $=12 \mathrm{~cm}$

\section{Usaremos varilla de 12 mm $@ 12$ cm}

\section{Armado del puente}

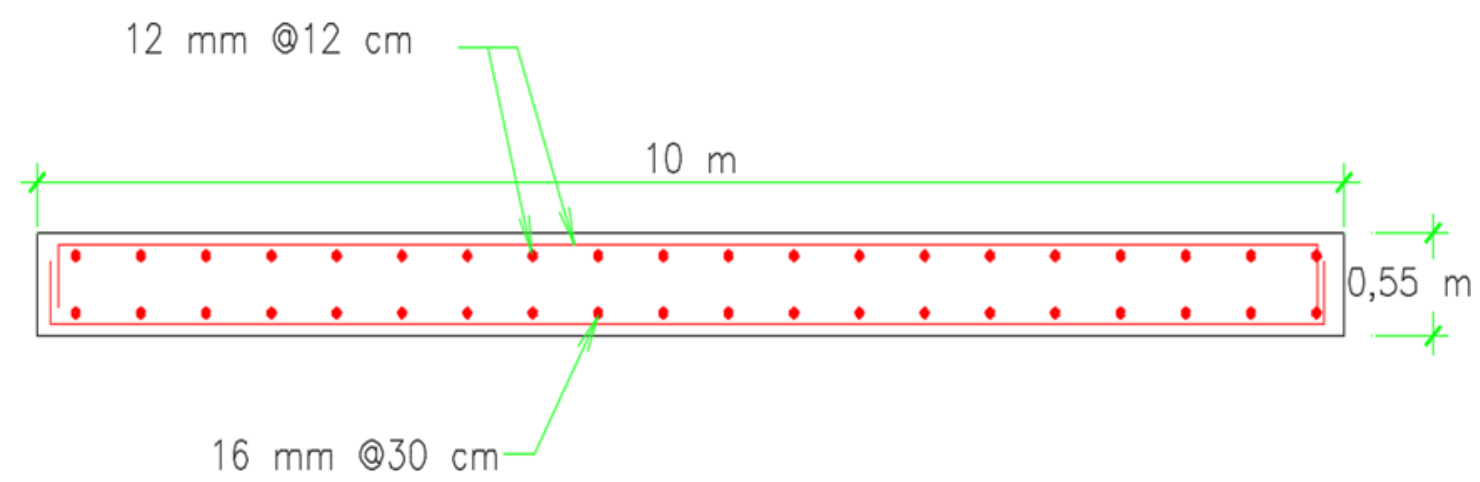

Figura 13. Armado Longitudinal 
Dom. Cien., ISSN: 2477-8818

Vol. 4, núm. 3, julio, 2018, pp. 189-210

Propuesta de puente aplicando el método de diseño AASHTO LRFD para la ciudad de Manta.

Fuente: Elaboración propia

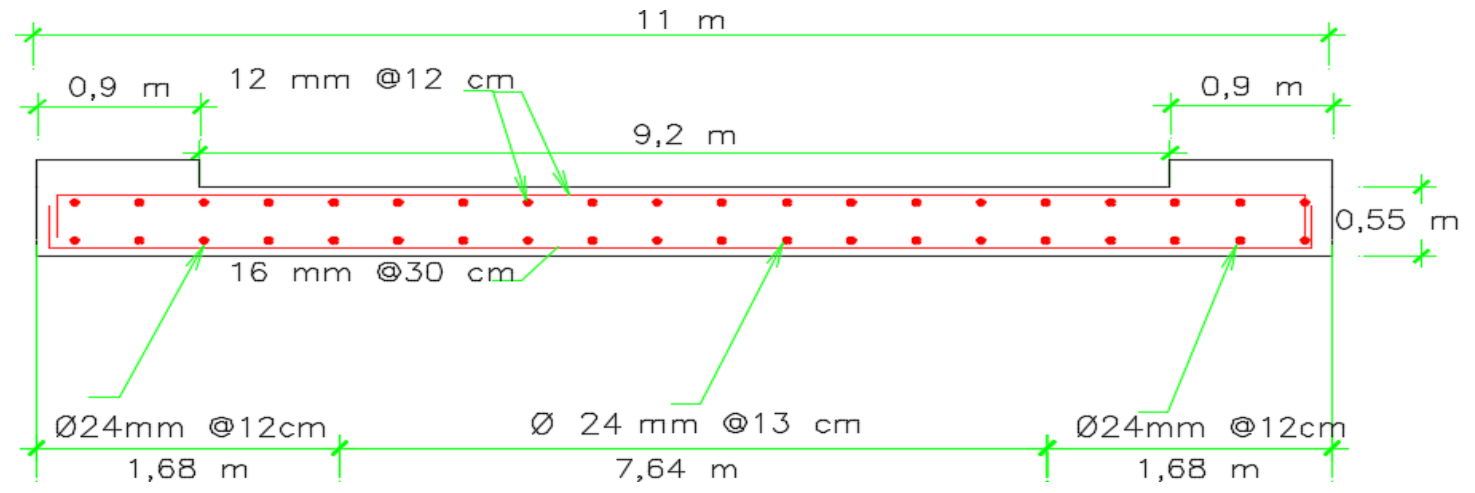

Figura 14. Armado Transversal

Fuente: Elaboración propia

\section{Plan de acción.}

Tabla 9. Plan de acción de la propuesta.

\begin{tabular}{|c|c|c|c|c|c|c|c|c|c|c|c|c|c|c|c|c|c|c|c|c|}
\hline \multirow[t]{2}{*}{ Actividades } & \multicolumn{4}{|c|}{ Mayo } & \multicolumn{4}{|c|}{ Junio } & \multicolumn{4}{|c|}{ Julio } & \multicolumn{4}{|c|}{ Agosto } & \multicolumn{4}{|c|}{ Septiembre } \\
\hline & 1 & 2 & 3 & 4 & 1 & 2 & 3 & 4 & 1 & 2 & 3 & 4 & 1 & 2 & 3 & 4 & 1 & 2 & 3 & 4 \\
\hline $\begin{array}{l}\text { Aplicación de } \\
\text { talleres } \\
\text { comportamiento y } \\
\text { seguridad } \\
\text { estructural de } \\
\text { puentes vehiculares }\end{array}$ & $\bar{x}$ & $x$ & & & & & & & & & & & & & & & & & & \\
\hline $\begin{array}{l}\text { Talleres de } \\
\text { capacitación sobre } \\
\text { la aplicación de los } \\
\text { métodos de diseño }\end{array}$ & & & $x$ & $x$ & $x$ & & & & & & & & & & & & & & & \\
\hline
\end{tabular}


Propuesta de puente aplicando el método de diseño AASHTO LRFD para la ciudad de Manta.

\begin{tabular}{|l|l|l|l|l|l|l|l|l|l|l|l|l|l|l|l|l|l|l|}
\hline $\begin{array}{l}\text { Socializar los } \\
\text { resultados del } \\
\text { análisis }\end{array}$ \\
$\begin{array}{l}\text { comparativos en los } \\
\text { talleres }\end{array}$
\end{tabular}

Recursos humanos: ingeniero de la localidad, autor de la propuesta, director de tesis, autoridades locales, cantonales y provinciales.

Recursos técnicos: cámara, encuestas, programas informáticos y equipo de diseño.

Evaluación. La evaluación de la propuesta constará de un seguimiento rutinario del cumplimiento de cada una de las actividades en el periodo establecido en el cual se evidenciará el grado de cumplimiento de cada actividad; el monitoreo mediante el cual se dispondrán de los recursos para la ejecución de las actividades, así como también se podrá medir el éxito de las mismas, es precisa la elaboración de un informe final a fin de documentar de manera adecuada la realización de la propuesta. 
Propuesta de puente aplicando el método de diseño AASHTO LRFD para la ciudad de Manta.

\section{Conclusiones}

Se analizó la información teórica referente a tema, por medio de la que se pudo identificar que la Norma AASHTO fue la primera norma reconocida para el diseño y la construcción de puentes, convirtiéndose rápidamente en una norma nacional, dentro de esta norma existe el método elástico (ASD) en el cual se debe estimar las cargas de trabajo o servicio, es decir las cargas que la estructura tiene que soportar, para luego diseñar los miembros estructurales con base en ciertos esfuerzos permisibles y existe el método plástico (LRFD) en este método las cargas de trabajo se estiman y se multiplican por ciertos factores de carga o de sobrecapacidad y los elementos estructurales se diseñan entonces con base en sus resistencias al colapso.

Por medio del proceso investigativo fue posible determinar que el correcto dimensionamiento de la estructura del puente losa, dependerá específicamente del proyecto que se esté llevando a cabo, considerando principalmente la longitud del puente, así como el peralte de la losa. Para la propuesta presentada se diseñó un puente losa de $10 \mathrm{~m}$ de longitud, $9.20 \mathrm{~m}$ de ancho y un peralte de losa de $0,55 \mathrm{~m}$, luego aplicando el procedimiento de cálculo y las formulas respectivas del método AASHTO LRFD el armado concluyó, que la distribución del acero principal para el borde interior es de varilla de 24 mm @ 13 cm, para el borde interior es de varilla de 24 mm 12 cm y para el acero de repartición es de varillas de 16 mm@30 cm

Se generó un análisis del método AASHTO LRFD, por medio del cual se pudo establecer como datos de mayor relevancia los momento últimos en la franja interior tanto en Resistencia como en Servicio, para el primer caso se obtuvo un momento de 213.55 T-m; mientras que para el segundo caso resultó un momento de $138.85 \mathrm{~T}-\mathrm{m}$ el diferencia que se presenta debido a que la AASHTO LRFD considera factores de 
Propuesta de puente aplicando el método de diseño AASHTO LRFD para la ciudad de Manta.

carga mayores en resistencia que en servicio y por lo tanto genera una mayor inversión técnica.

\section{Referencias Bibliográficas}

AASHTO. (1996). Standard Specifications for highway bridge. Washington: 16th Ed.

AASHTO. (2004). Especificaciones para el diseño de puentes por el método LRFD. Washington:

3th. Ed.

Escalante Cervera, V. (2014). Diseño de estructuras metálicas LRFD. Santo Domingo - República

Dominicana: Editorial Independiente.

FELIX, D. (2015). Diseño y análisis de la superestructura de un puente de hormigón. Guayaquil:

Universidad Laica Vicente Rocafuerte.

Nilson, A. (2011). Diseño de estructuras de concreto. Bogotá - Colombia: Graw Hill.

OCHOA, A. (2008). Diseño de superestructura de puentes de hormigón armado. Valdivia:

Universidad Austral de Chile.

Perales, A. (2013). Diseño automatizado de vigas metálicas para puentes carreteros. Chile:

Universidad Austral de Chile. 